\title{
Synthetic Approaches and Biological Evaluation of Some New Sulfonate Ester-Containing Imidazolone Derivatives
}

\author{
Osman M. O. Habib, Hussein M. Hassan, Evelin B. Moawad, Ahmed El-Mekabaty* \\ Department of Chemistry, Faculty of Science, Mansoura University, Mansoura 35516, Egypt
}

\begin{abstract}
Oxazolone derivative 2 was utilised as a key intermediate for the synthesis of some new imidazolone derivatives. Reaction of $\mathbf{2}$ with $p$-aminoacetophenone in the presence of acetic acid containing catalytic amount of freshly fused sodium acetate gives the corresponding imidazolone derivative $\mathbf{3}$ which on reaction with diethyl oxalate in the presence of granulated sodium metal afforded the corresponding imidazolone derivative 4. Condensation of compound 3 with some aromatic aldehydes afforded the corresponding cinnamoylphenyl derivatives $\mathbf{5}_{\mathrm{a}-\mathrm{c}}$, respectively. Besides, compounds $\mathbf{5}_{\mathrm{a}-\mathrm{c}}$ reacted with hydrazine hydrate in boiling ethanol and yield the corresponding 4-arylidene-1- ( $p$-(5-aryl-2- pyrazolin-3-y1) phenyl)-2-phenyl-2-imidazolin-5-ones $\mathbf{6}_{\mathbf{a}-\mathrm{c} \text {, }}$,espectively. Moreover, refluxing of compounds $\mathbf{5}$ a-c with both ethylcyanoacetate and/or malononitrile in ethanol and ammonium acetate afforded $\mathbf{7}_{\mathrm{a}-\mathrm{c}}$ and $\mathbf{8}_{\mathrm{a}-\mathrm{c}}$, respectively. Claisen reaction of $\mathbf{3}$ with ethylacetate gives the corresponding imidazolone derivative 9 which on reaction with thiourea in ethanol afforded the corresponding thienopyrimidine derivative 10. On the other hand, reaction of oxazolone $\mathbf{2}$ with both ethyl $p$-aminobenzoate and anthranilic acid in glacial acetic acid and freshly fused sodium acetate has been also studied. Structures of the newly synthesized compounds were established by elemental analysis and spectral data. Representative compounds of the synthesized products were tested and evaluated as antifungal and antimicrobial agents. Most of compounds exhibited good activities, where $\mathbf{3}, \mathbf{5}_{\mathbf{b}}, \mathbf{5}_{\mathfrak{c}}, \mathbf{6}_{\mathbf{a}}, \mathbf{7}_{\mathbf{b}}, \mathbf{7}_{\mathfrak{c}}, \mathbf{8}_{\mathbf{a}}, \mathbf{9}, \mathbf{1 1}, \mathbf{1 3}$ and $\mathbf{1 4}$ exhibited good activities against Bacillus Thuringenesis and Klebseilla Pneumonia, while compounds $3, \mathbf{4}, \mathbf{6}_{\mathbf{a}}, \mathbf{6}_{\mathbf{b}}, \mathbf{6}_{\mathfrak{c}}, \mathbf{7}_{\mathbf{c}}, \mathbf{8}_{\mathbf{a}}, \mathbf{8}_{\mathbf{c}}, \mathbf{1 1}, \mathbf{1 2}$ and $\mathbf{1 6}$ exhibited good activities against Trichoderma Herzianum and Trichoderma Virdi.
\end{abstract}

Keywords Oxazolone, Imidazolone, Pyrazole, Pyrimidine, Antibacterial

\section{Introduction}

Substituted oxazolone and imidazolone derivatives have become of great importance due to their wide range of biological activity. Previous studies have been reported that, it exhibit good anticonvulsant, bactericidal, fungicidal and insecticidal activities. Besides, they were shown a wide range of pharmaceutical properties [1-5]. On the other hand, it has been stated that, compounds containing aromatic sulfonate or sulfonamide moieties possess high acaricidal as well as insecticidal activity [6-7].

\section{Results and Discussion}

The present investigation aims to synthesize a series of products bearing both aryl sulfonate, oxazolone and imidazolone moieties in the same molecule hoping that these new products might show high biocidal activity. Thus, The

* Corresponding author:

a_el_m11@yahoo.com (Ahmed El-Mekabaty)

Published online at http://journal.sapub.org/ajoc

Copyright (C) 2012 Scientific \& Academic Publishing. All Rights Reserved required $(Z)-4-((5-$ oxo-2-phenyloxazol-4(5H)-ylidene) methyl)phenyl-4-methyl benzenesulfonate 2 , was prepared by means of the reaction of 4-toluenesulfonyloxy benzaldehyde 1 with hippuric acid and acetic anhydride in the presence of freshly fused sodium acetate according to the method reported in literature [8], (scheme 1).

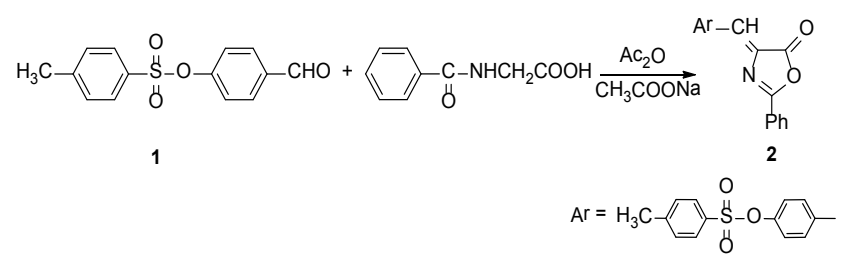

Scheme 1. Synthesis of oxazolone derivative 2

In the present study, oxazolone 2 reacts with $p$ - aminoacetophenone in glacial acetic acid and fused sodium acetate giving (Z)-4-((1-(4-acetylphenyl)-5-oxo-2-phenyl -1 $H$-imidazol-4(5H)-ylidene)methyl)phenyl4-methylbenzen e sulfonate 3. Structure of $\mathbf{3}$ was confirmed by both analytical and spectral data. The IR spectrum exhibited absorption bands at 1715 and $1679 \mathrm{~cm}^{-1}$ characteristic for $\left(-\mathrm{COCH}_{3}\right)$ and $(\mathrm{CON})$ groups, respectively. ${ }^{1} \mathrm{H}-\mathrm{NMR}$ spectrum exhibited singlet signal at $\delta 2.6$ characteristic for $\mathrm{COCH}_{3}$ protons. Moreover, the mass spectrum measurement gave an evi- 
dence for the proposed structure, which showed the molecular ion peak at $\mathrm{m} / \mathrm{e} 536\left(\mathrm{M}^{+}\right)$(Scheme 2).

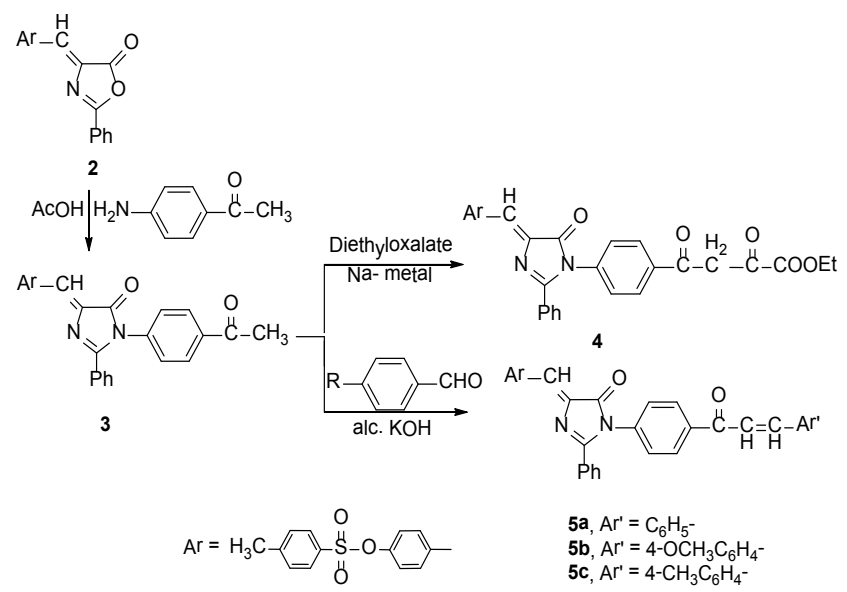

Scheme 2. Reaction of oxazolone 2 with $p$-aminoacetophenone

Treatment of compound $\mathbf{3}$ with diethyl oxalate in the presence of granulated sodium metal produced the corresponding imidazolone derivative $\mathbf{4}$. In addition, compound $\mathbf{3}$ undergoes condensation with some aromatic aldehydes namely, benzaldehyde; $p$-anisaldehyde and $p$-tolualdehyde to give the corresponding cinnamoylphenyl derivatives $\mathbf{5}_{\mathbf{a}-\mathrm{c}}$, respectively (scheme 2). Chemical structures of compounds $\mathbf{5}_{\text {a-c }}$ were established on both elemental and spectral evidences. So, the IR spectrum in general showed stretching frequencies at 1675 and $1645 \mathrm{~cm}^{-1}$ attributable to the CON and $\mathrm{C}=\mathrm{O}$ (Chalcone) groups. ${ }^{1} \mathrm{H}-\mathrm{NMR}$ spectra of compound $\mathbf{5}_{\mathbf{b}}$ showed multiple signals at about $\delta 8.2-8.3$ corresponding to olefinic $\mathrm{CH}=\mathrm{CH}$ protons and singlet signal at $\delta 4.4$ for the
$\mathrm{OCH}_{3}$ protons. Besides, compound $\mathbf{5}_{\mathbf{c}}$ revealed multiple signals at about $\delta 8.2-8.3$ corresponding to olefinic $\mathrm{CH}=\mathrm{CH}$ protons and singlet signal at $\delta 2.1$ for the $-\mathrm{CH}_{3}$ protons. Moreover, structures $\mathbf{5}_{\mathrm{a}-\mathrm{c}}$ were confirmed by the mass spectral measurements which showed the molecular ion peaks at $\mathrm{m} / \mathrm{e} 624\left(\mathrm{M}^{+}\right), \mathrm{m} / \mathrm{e} 654\left(\mathrm{M}^{+}\right)$and $\mathrm{m} / \mathrm{e} 638\left(\mathrm{M}^{+}\right)$, respectively.

Besides, compounds $\mathbf{5}_{\mathrm{a}-\mathrm{c}}$ reacted with hydrazine hydrate in boiling ethanol to give the corresponding 4-arylidene-1( $p$-(5-aryl-2-pyrazolin-3-y1)phenyl)-2-phenyl-2-imidazolin5 -ones $\mathbf{6}_{\mathrm{a}-\mathrm{c}}$, respectively. Structures $\mathbf{6}_{\mathrm{a}-\mathrm{c}}$ were established on both elemental and spectral evidences. So, ${ }^{1} \mathrm{H}-\mathrm{NMR}$ spectrum in general showed revealed doublet signals at $\delta 3.1$ corresponding for $\mathrm{CH}_{2}$ protons of pyrazole. The IR spectra of these compounds showed characteristic absorption bands at $3200-3260 \mathrm{~cm}^{-1}$ attributable to (-NH) group. In addition, structures $\mathbf{6}_{\mathbf{a}-\mathrm{c}}$ were confirmed by the mass spectrophotometric measurements which showed the molecular ion peaks at m/e $638\left(\mathrm{M}^{+}\right)$, m/e $668\left(\mathrm{M}^{+}\right)$and $\mathrm{m} / \mathrm{e} 652\left(\mathrm{M}^{+}\right)$, respectively. Moreover, refluxing of compounds $\mathbf{5}_{\mathrm{a}-\mathrm{c}}$ with both ethylcyanoacetate and/or malononitrile in ethanol and excess of ammonium acetate afforded $\mathbf{7}_{\mathrm{a}-\mathrm{c}}$ and $\mathbf{8}_{\mathbf{a}-\mathrm{c}}$, respectively (scheme 3 ).

On the other hand, treatment of imidazolone 3 with ethylacetate in the presence of sodium metal gives the corresponding 1,3-diketone derivative 9 which reacted with thiourea in ethanol giving the thiopyrimidine derivative $\mathbf{1 0}$. The structure of compound $\mathbf{1 0}$ was elucidated on the basis of both analytical and spectral data. The IR spectrum of compound 10 displayed absorption band at $1370 \mathrm{~cm}^{-1}$ due to $(\mathrm{C}=\mathrm{S})$ group. In addition, the mass spectrum gives a molecular ion peak at m/e $621\left(\mathrm{M}^{+}+2\right)$ (scheme 4$)$.

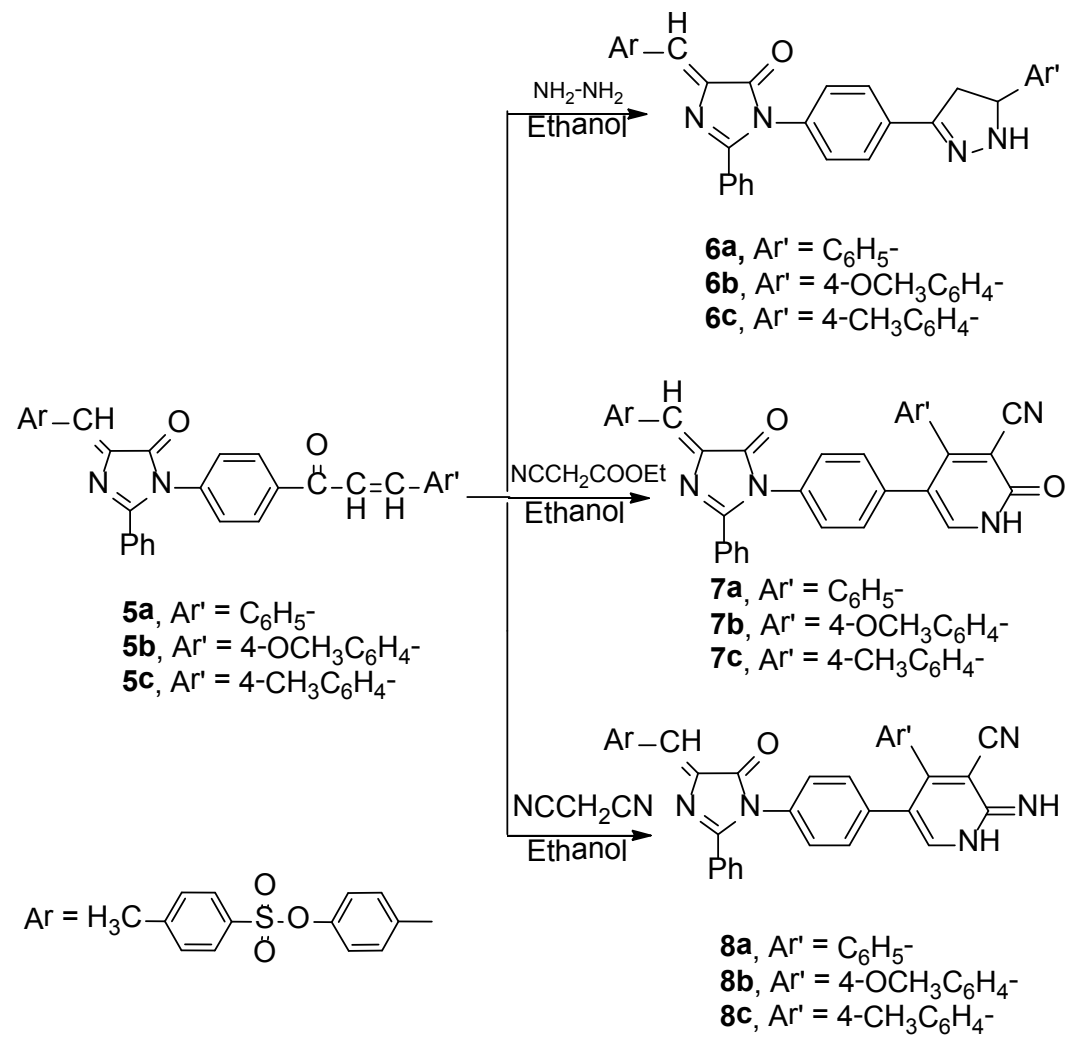

Scheme 3. Reaction of imidazolone derivatives 5a-c with hydrazine hydrate, ethylcyanoacetate and malononitrlie 
Oxazolone 2 reacted with ethyl $p$-aminobenzoate in glacial acetic acid and freshly fused sodium acetate to give (Z)-ethyl 4-(5-oxo-2-phenyl-4-(4-(tosyloxy)benzylidene)-4, 5-dihydro-1H-imidazol-1-yl) benzoate 11. The IR spectrum of 11 showed absorption bands at 1680 and $1725 \mathrm{~cm}^{-1}$ due to $(\mathrm{CON})$ and carbonyl ester groups. ${ }^{1} \mathrm{H}-\mathrm{NMR}$ showed signals at $\delta$ 4.39-4.42 (q, $\left.2 \mathrm{H},-\mathrm{CH}_{2} \mathrm{CH}_{3}\right)$ and $\delta$ 1.39-1.43 (t, 3H, $\left.-\mathrm{CH}_{2} \mathrm{CH}_{3}\right)$. The mass spectrum of compound $\mathbf{1 1}$ showed the molecular ion peak at $\mathrm{m} / \mathrm{e} 566\left(\mathrm{M}^{+}\right)$. Tratment of imidazolone 11 with benzylamine in ethanol afforded (Z)-4-((1-(4(benzylcar bamoyl)phenyl)-5-oxo-2-phenyl-1H-imidazol-4( $5 H$ )-ylidene)methyl)phenyl4-methylbenzenesulfonate 12, (scheme 5). The structure of compound $\mathbf{1 2}$ was established on both analytical and spectral data. The IR spectrum exhibited bands at 1690 and $3478 \mathrm{~cm}^{-1}$ corresponding to -CON and $-\mathrm{NH}$ groups and revealed the absence of a carbonyl of ester group absorption band. ${ }^{1} \mathrm{H}-\mathrm{NMR}$ spectrum revealed singlet signals at $\delta 4.2$ and $\delta 11$ characteristic for $-\mathrm{CH}_{2} \mathrm{Ph}$. The mass spectrum revealed molecular ion peak at $\mathrm{m} / \mathrm{e} 626.6$ $\left(\mathrm{M}^{+}-1\right)$ with relative abundance corresponding to the molecular formula $\mathrm{C}_{37} \mathrm{H}_{29} \mathrm{~N}_{3} \mathrm{O}_{5} \mathrm{~S}$.

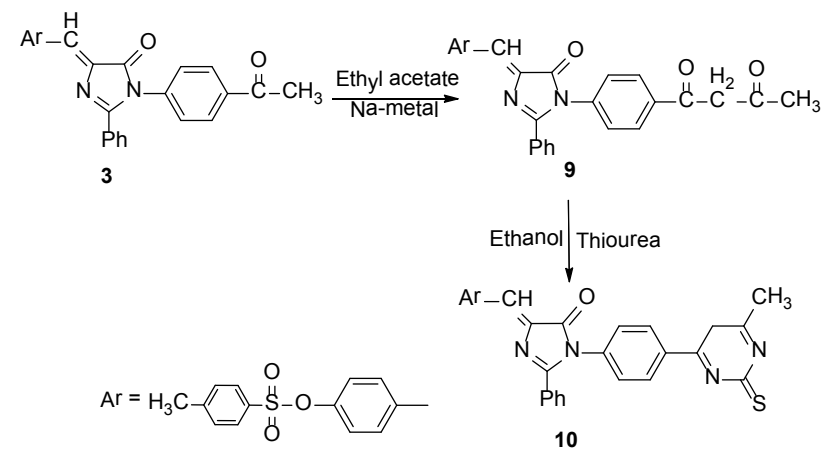

Scheme 4. Reaction of imidazolone derivative 3 with ethyl acetate

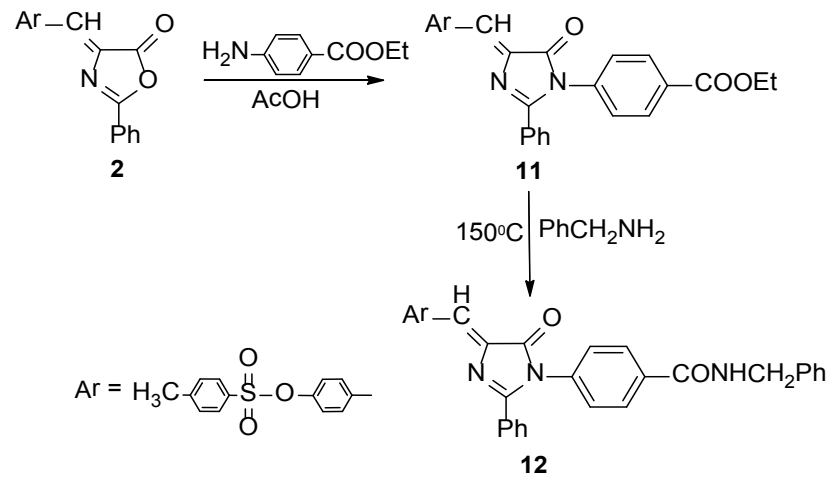

Scheme 5. Condensation of oxazolone 2 with ethyl $p$-aminobenzoate

Furthermore, the reaction of imidazolone 11 with hydrazine hydrate is interesting since refluxing in ethanol gives four products 13-16 which were isolated by using column chromatography (scheme 6). Formation of compound $\mathbf{1 4}$ could proceed via the plausible mechanism, (scheme 7).

On the other hand, refluxing of oxazolone 2 with anthranilic acid in glacial acetic acid and fused sodium acetate gives the benzoxazinone derivative $\mathbf{1 7}$ instead of compound 17 ' (scheme 8). The structure of compound $\mathbf{1 7}$ was elucidated on the basis of both analytical and spectral data. The IR spectrum showed absorption bands at 3470, 1760 and 1595 $\mathrm{cm}^{-1}$ due to amidic $(\mathrm{NH})$, carbonyl of lactone and $(\mathrm{C}=\mathrm{C})$ groups. ${ }^{1} \mathrm{H}-\mathrm{NMR}$ spectrum revealed singlet signals at $\delta 4.9$ characteristic for $\mathrm{CH}=\mathrm{C}$ protons. Moreover, the mass spectrum showed the molecular ion peak at $\mathrm{m} / \mathrm{e} 538\left(\mathrm{M}^{+}\right)$. Formation of compound $\mathbf{1 7}$ could proceed via the plausible mechanism (scheme 9).

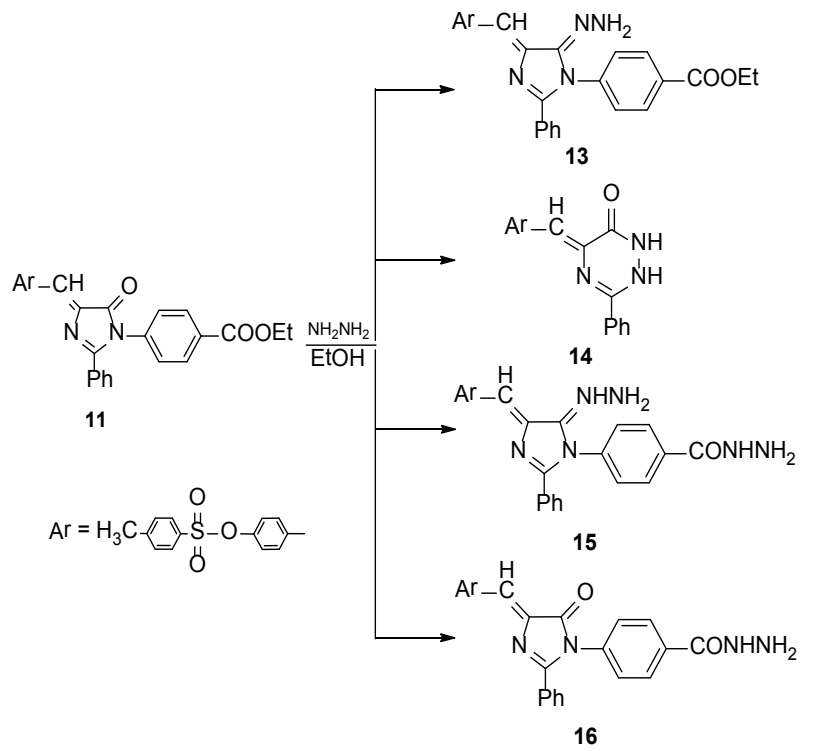

Scheme 6. Reaction of imidazolone derivative $\mathbf{1 1}$ with hydrazine hydrate

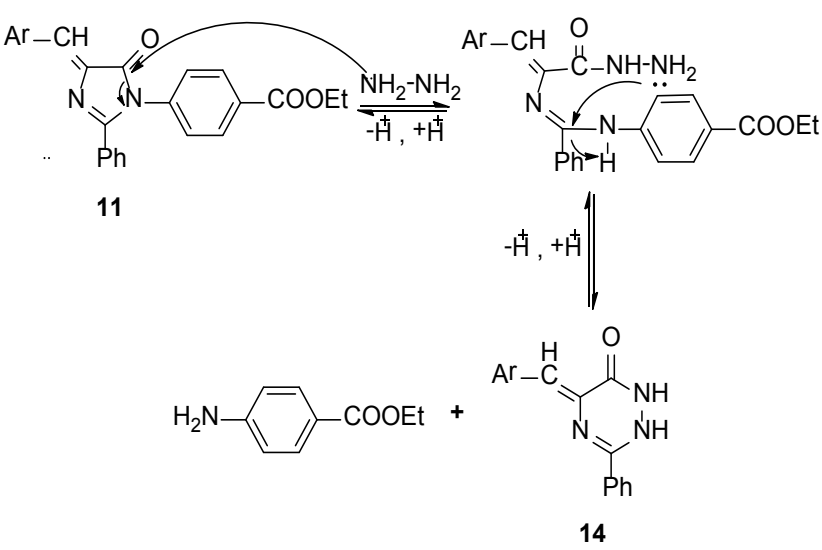

Scheme 7. The plausible mechanism for the formation of compound $\mathbf{1 4}$

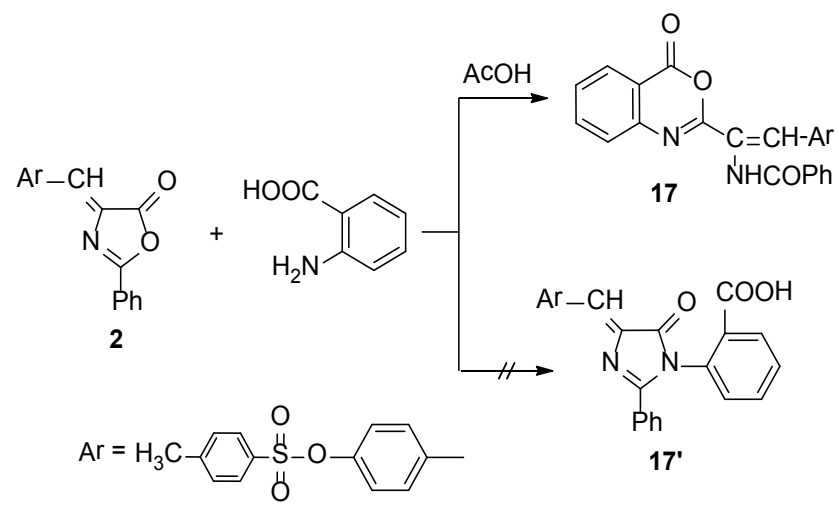

Scheme 8. Condensation of oxazolone 2 with anthranilic acid 


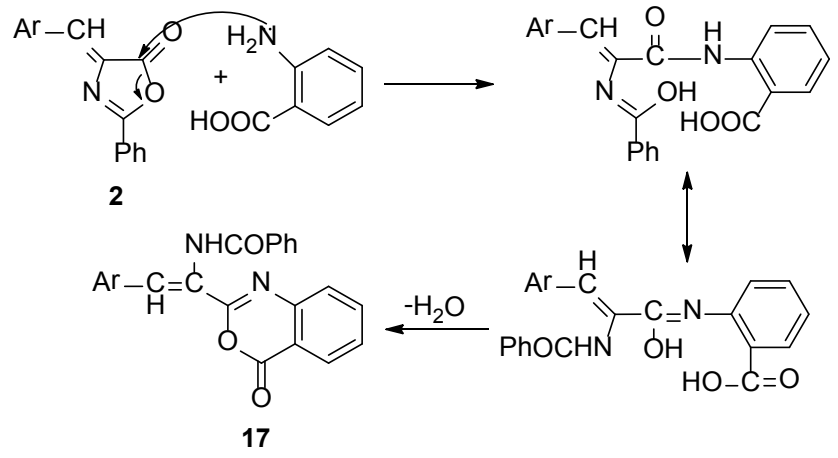

Scheme 9. The plausible mechanism for the formation of compound $\mathbf{1 7}$

\section{Antimicrobial Activity}

All the compounds were subjected to biological screening and they showed promising antibacterial and antifungal activity which were comparable to the activity of known standard drugs. The results are summarized in Table $(1,2)$. The results for antibacterial activities depicted in Table 1 revealed that, most compounds exhibited good activities against the reference chemotherapeutics, where $\mathbf{3}, \mathbf{5 b}, \mathbf{5 c}, \mathbf{6 a}$, 7b, 7c, 8a, 9, 11, 13 and 14 exhibited good activities against Bacillus Thuringenesis and compounds $3, \mathbf{5 a}, \mathbf{5 c}, \mathbf{6 a}, 7 \mathbf{b}, \mathbf{8 a}$, 10, 13, 14, 15 and 16 exhibited good activities against Klebseilla Pneumonia. On the other hand, the results for antifungal activities depicted in Table 2 revealed that, compounds 3, 4, 6a, 6b, 6c, 7c, 8a, 8c, 11, 12 and 16 exhibited good activities against the Trichoderma Herzianum and Trichoderma Virdi. Therefore, the obtained results indicated that, the antimicrobial activity is dependent on the attached groups with oxazolone and imidazolone derivatives. A comparison of antibacterial and antifungal activities of compounds with their structures revealed that, the compounds that bearing aryl sulfonate, oxazolone and imidazolone moieties in the same molecule exhibited significant activity against Bacillus Thuringenesis, Klebseilla Pneumonia, Trichoderma Herzianum and Trichoderma Virdi.

\section{Experimental}

All melting points (uncorrected) were determined on Gallenkamp electric melting point apparatus, FTIR spectra ( $\mathrm{KBr}$ disk) were recorded on a Nicolet Magna. IR model 550 spectrophotometers, ${ }^{1} \mathrm{H}-\mathrm{NMR}$ spectra, were determined on Brucker Wpsy 300 MHZ spectrometer with TMS as internal standard and the chemical shifts are in $\sigma \mathrm{ppm}$. Mass spectra were recorded at $70 \mathrm{ev}$ with a Varian MAT 311. Elemental analyses are satisfactory for all synthesized compounds (2-17), all analyses were carried out in Faculty of Science, Cairo University, Egypt. (Z)-4-((5-oxo-2-phenyloxazol4(5H)-ylidene)methyl)phenyl-4-methylbenzene sulfonate 2 was prepared previously as shown in literature [8].

Synthesis of (Z)-4-((1-(4-acetylphenyl)-5-oxo-2-phenyl -1H-imidazol-4(5H)-ylidene)methyl)phenyl-4-methyl benzenesulfonate (3).

A mixture of oxazolone $2(0.01 \mathrm{~mole})$ and $p$-amino acetophenone $(0.01 \mathrm{~mole})$ in glacial acetic acid $(30 \mathrm{ml})$ containing freshly fused sodium acetate $(0.5 \mathrm{gm})$ was heated under reflux for 8 hours. The reaction mixture was concentreated under reduced pressure and the separated solid product was filtered off, and recrystallized from acetic acid to give 3 .

Yellow crystals; Yield 45\%; m.p. $180-182^{\circ} \mathrm{C}$; IR (KBr): $v / \mathrm{cm}^{-1}: 1715\left(\mathrm{COCH}_{3}\right), 1679(\mathrm{CO}$, amidic $), 1645(\mathrm{C}=\mathrm{N})$, $1360\left(\mathrm{SO}_{3}\right)$; $\operatorname{EIMS}(\mathrm{m} / \mathrm{z})(\%): 536\left(\mathrm{M}^{+}, 21.7\right), 419(2.1), 380$ (25.4), 278 (9.8), 222 (86), 155 (13), 117 (32), 91 (100), 65 (47.7); ${ }^{1} \mathrm{H}$ NMR (DMSO) $(\delta, \mathrm{ppm}), 2.4\left(\mathrm{~s}, 3 \mathrm{H}, \mathrm{CH}_{3}\right), 2.6(\mathrm{~s}$, $\left.3 \mathrm{H}, \mathrm{COCH}_{3}\right), 7.1-8.2(\mathrm{~m}, 18 \mathrm{H}, \mathrm{Ar}-\mathrm{H}, \mathrm{CH}=\mathrm{C})$; Anal. for $\mathrm{C}_{31} \mathrm{H}_{24} \mathrm{~N}_{2} \mathrm{O}_{5} \mathrm{~S}$ (536.6): Calcd.: C, 69.39; H, 4.51; N, 5.22\%; Found: C, 69.33; H, 4.49; N, 5.19\%.

Table 1. Diameter of inhibited zones (I. Z. D.) in millimeters as a criterion of antibacterial activity of the synthesized compounds at a concentration of 10 $\mathrm{mg} / \mathrm{ml}$

\begin{tabular}{|c|c|c|c|c|c|}
\hline \multirow{3}{*}{ Comp No. } & \multicolumn{2}{|c|}{ Bacteria } & \multirow{3}{*}{ Comp No. } & \multicolumn{2}{|c|}{ Bacteria } \\
\hline & Bacillus Thuringenesis & Klebseilla Pneumonia & & Bacillus Thuringenesis & Klebseilla Pneumonia \\
\hline & I. Z.D. mm & I. Z.D. mm & & I. Z.D. $\mathrm{mm}$ & I. Z.D. $\mathrm{mm}$ \\
\hline 2 & 27 & 33 & $8 \mathrm{c}$ & 28 & 28 \\
\hline 3 & 30 & 36 & 9 & 31 & 21 \\
\hline 4 & 29 & 30 & 10 & 18 & 38 \\
\hline $5 \mathbf{a}$ & 29 & 44 & 11 & 30 & 33 \\
\hline $5 \mathbf{b}$ & 32 & 33 & 12 & 29 & 28 \\
\hline $5 \mathrm{c}$ & 35 & 45 & 13 & 31 & 36 \\
\hline $6 \mathbf{a}$ & 39 & 50 & 14 & 41 & 41 \\
\hline $6 \mathbf{b}$ & 24 & 29 & 15 & 26 & 39 \\
\hline $6 \mathbf{c}$ & 29 & 34 & 16 & 24 & 44 \\
\hline $7 \mathbf{a}$ & 19 & 28 & 17 & 31 & 31 \\
\hline $7 \mathbf{b}$ & 32 & 35 & Flummox & 24 & 29 \\
\hline $7 \mathrm{c}$ & 35 & 26 & Ampicillin & 29 & 34 \\
\hline $8 \mathbf{a}$ & 39 & 45 & Chloramphenicol & 19 & 28 \\
\hline $8 \mathbf{b}$ & 24 & 29 & - & - & - \\
\hline
\end{tabular}


Table 2. Diameter of inhibited zones (I. Z. D.) in millimeters as a criterion of antifungal activity of the synthesized compounds at a concentration of 10 $\mathrm{mg} / \mathrm{ml}$

\begin{tabular}{|c|c|c|c|c|c|}
\hline \multirow{3}{*}{ Comp No. } & \multicolumn{2}{|c|}{ Fungi } & \multirow{3}{*}{ Comp No. } & \multicolumn{2}{|c|}{ Fungi } \\
\hline & Trichoderma Herzianum & Trichoderma Virdi & & Trichoderma Herzianum & Trichoderma Virdi \\
\hline & I. Z.D. $\mathrm{mm}$ & I. Z.D. $\mathrm{mm}$ & & I. Z.D. $\mathrm{mm}$ & I. Z.D. $\mathrm{mm}$ \\
\hline 2 & 31 & 41 & $8 c$ & 35 & 45 \\
\hline 3 & 35 & 46 & 9 & 41 & 32 \\
\hline 4 & 40 & 41 & 10 & 37 & 38 \\
\hline $5 \mathbf{5 a}$ & 41 & 36 & 11 & 44 & 36 \\
\hline $5 \mathbf{b}$ & 38 & 33 & 12 & 49 & 40 \\
\hline $5 c$ & 37 & 26 & 13 & 46 & 47 \\
\hline $6 a$ & 44 & 39 & 14 & 39 & 40 \\
\hline $6 b$ & 49 & 36 & 15 & 24 & 39 \\
\hline $6 c$ & 46 & 49 & 16 & 29 & 45 \\
\hline $7 a$ & 29 & 44 & 17 & 19 & 38 \\
\hline $7 b$ & 31 & 48 & Flummox & 35 & 41 \\
\hline $7 c$ & 48 & 26 & Ampicillin & 41 & 36 \\
\hline $8 \mathbf{8}$ & 46 & 39 & Chloramphenicol & 32 & 31 \\
\hline $8 b$ & 40 & 36 & - & - & - \\
\hline
\end{tabular}

Synthesis of (Z)-ethyl2,4-dioxo-4-(4-(5-oxo-2-phenyl-4(4-(tosyloxy)benzylidene)-4,5-dihydro-1H-imidazol-1-yl) phenyl)butanoate (4).

A mixture of 3 ( 0.05 mole), dry diethyloxalate $(1.15 \mathrm{~mole})$ and granulated sodium metals $(0.1$ mole) were heated at $150^{\circ} \mathrm{C}$ for 7 hours. The mixture was triturated with ethanol, acidified with dil. $\mathrm{HCl}$ and the separated solid was recrystallized from ethanol to give 4.

Brown powder; Yield 25\%; m.p. $250-252^{\circ} \mathrm{C}$; IR ( $\left.\mathrm{KBr}\right)$ : v/ $\mathrm{cm}^{-1}: 1750$ (CO, ester), 1720 (CO), 1670 (CO, amidic), $1640(\mathrm{C}=\mathrm{N})$ and $1360\left(\mathrm{SO}_{3}\right)$; $\operatorname{EIMS}(\mathrm{m} / \mathrm{z})(\%): 636\left(\mathrm{M}^{+}, 27\right)$, 375 (54), 272 (45), 261 (31), 233 (21), 118 (100), $70(68) ;{ }^{1} \mathrm{H}$ NMR (DMSO) $(\delta, \mathrm{ppm}), 2.4\left(\mathrm{~s}, 3 \mathrm{H}, \mathrm{CH}_{3}\right), 3.71-3.74(\mathrm{q}, 2 \mathrm{H}$, $\left.\mathrm{CH}_{2}\right), 1.25-1.27\left(\mathrm{t}, 3 \mathrm{H}, \mathrm{CH}_{3}\right), 4.7\left(\mathrm{~s}, 2 \mathrm{H}, \mathrm{CH}_{2}\right), 7.1-8.2(\mathrm{~m}$, $18 \mathrm{H}, \mathrm{Ar}-\mathrm{H}, \mathrm{CH}=\mathrm{C})$; Anal. for $\mathrm{C}_{35} \mathrm{H}_{28} \mathrm{~N}_{2} \mathrm{O}_{8} \mathrm{~S}$ (636.6): Calcd.: C, 66.03; H, 4.43; N, 4.4\%; Found: C, 66.0; H, 4.40; N, $4.2 \%$.

Synthesis of 4-((1Z)-(5-oxo-2-phenyl-1-(4-(3- arylacryloyl)phenyl)-1 $H$-imidazol-4(5H)-ylidene)methyl)phenyl 4-methylbenzenesulfonate $\left(5_{\mathrm{a}-\mathrm{c}}\right)$.

General procedure

A mixture of $3(0.01 \mathrm{~mole})$ and an appropriate aldehydes (0.01mole) in ethanolic $\mathrm{KOH}$ was refluxed for 8-11 hours. The solids that separated on acidification with dil. $\mathrm{HCl}$ were filtered and recrystallized from acetone to give $\mathbf{5}_{\text {a-c }}$.

4-((1Z)-(5-oxo-2-phenyl-1-(4-(3-phenylacryloyl)phenyl)$1 H$-imidazol-4(5H)-ylidene)methyl)phenyl-4-methylbenz enesulfonate $5_{\mathrm{a}}$ :

Yellow sheets; Yield 33\%; m.p. 211-213 ${ }^{\circ} \mathrm{C}$; IR (KBr): $v / \mathrm{cm}^{-1}: 1675$ (CO, amidic), 1645 (CO, chalcone), 1610 $(\mathrm{C}=\mathrm{N}), 1360\left(\mathrm{SO}_{3}\right), 1599(\mathrm{C}=\mathrm{C})$; EIMS $(\mathrm{m} / \mathrm{z})(\%): 624\left(\mathrm{M}^{+}\right.$, 34), 503 (71), 328 (18), 266 (10), 183 (100), 112 (80), 70 (50); ${ }^{1} \mathrm{H}$ NMR (DMSO) ( $\delta$, ppm), 2.4 (s, 3H, $\left.\mathrm{CH}_{3}\right), 8.26-8.36$ (m, $2 \mathrm{H}, \mathrm{CH}=\mathrm{CH}), 7.1-8.1(\mathrm{~m}, 23 \mathrm{H}, \mathrm{Ar}-\mathrm{H}, \mathrm{CH}=\mathrm{C})$; Anal. for $\mathrm{C}_{38} \mathrm{H}_{28} \mathrm{~N}_{2} \mathrm{O}_{5} \mathrm{~S}$ (624.7): Calcd.: C, 73.06; H, 4.52; N, 4.48\%; Found: C, 72.98; H, 4.49; N, 4.46\%.

4-((1Z)-(1-(4-(3-(4-methoxyphenyl)acryloyl)phenyl)-5-ox o-2-phenyl-1H-imidazol-4(5H)-ylidene)methyl)phenyl-4- methylbenzenesulfonate $\mathbf{5}_{\mathbf{b}}$ :

Yellow sheets; Yield 45\%; m.p. $177-179^{\circ} \mathrm{C}$; IR (KBr): $\mathrm{v} / \mathrm{cm}^{-1}: 1675$ (CO, amidic), 1645 (CO, chalcone), 1610 $(\mathrm{C}=\mathrm{N}), 1360\left(\mathrm{SO}_{3}\right), 1599(\mathrm{C}=\mathrm{C})$; $\operatorname{EIMS}(\mathrm{m} / \mathrm{z})(\%): 654\left(\mathrm{M}^{+}\right.$, 13), 566 (55), 460 (13), 312 (21), 108 (33), 91 (100), 65 (18); ${ }^{1} \mathrm{H}$ NMR (DMSO) $(\delta, \mathrm{ppm}), 2.4\left(\mathrm{~s}, 3 \mathrm{H}, \mathrm{CH}_{3}\right), 4.4(\mathrm{~s}, 3 \mathrm{H}$, $\left.\mathrm{OCH}_{3}\right), 8.26-8.36(\mathrm{~m}, 2 \mathrm{H}, \mathrm{CH}=\mathrm{CH}), 7.1-8.1(\mathrm{~m}, 23 \mathrm{H}, \mathrm{Ar}-\mathrm{H}$, $\mathrm{CH}=\mathrm{C}$ ); Anal. for $\mathrm{C}_{39} \mathrm{H}_{30} \mathrm{~N}_{2} \mathrm{O}_{6} \mathrm{~S}$ (654.7): Calcd.: C, 71.54; $\mathrm{H}$, 4.62 , N, 4.27\%; Found: C, 71.49; H, 4.59, N, 4.21\%.

4-((1Z)-(5-oxo-2-phenyl-1-(4-(3-(p-tolyl)acryloyl)phenyl) -1H-imidazol-4(5H)-ylidene)methyl)phenyl-4-methyl benzenesulfonate $\mathbf{5}_{\boldsymbol{c}}$ :

Yellow sheets; Yield 30\%; m.p. $154-156^{\circ} \mathrm{C}$; IR (KBr): $v / \mathrm{cm}^{-1}: 1675$ (CO, amidic), 1645 (CO, chalcone), 1610 $(\mathrm{C}=\mathrm{N}), 1360\left(\mathrm{SO}_{3}\right), 1599(\mathrm{C}=\mathrm{C})$; $\operatorname{EIMS}(\mathrm{m} / \mathrm{z})(\%): 638\left(\mathrm{M}^{+}\right.$, 8.3), 480 (61), 348 (100), 220 (31), 160 (54), 90 (30), 70 (61); ${ }^{1} \mathrm{H}$ NMR (DMSO) $(\delta, \mathrm{ppm}), 2.4\left(\mathrm{~s}, 3 \mathrm{H}, \mathrm{CH}_{3}\right), 2.1(\mathrm{~s}, 3 \mathrm{H}$, $\left.\mathrm{CH}_{3}\right), 8.26-8.36(\mathrm{~m}, 2 \mathrm{H}, \mathrm{CH}=\mathrm{CH}), 7.1-8.1(\mathrm{~m}, 23 \mathrm{H}, \mathrm{Ar}-\mathrm{H}$, $\mathrm{CH}=\mathrm{C}$ ); Anal. for $\mathrm{C}_{39} \mathrm{H}_{30} \mathrm{~N}_{2} \mathrm{O}_{5} \mathrm{~S}$ (638.7): Calcd.: C, 73.34; $\mathrm{H}$, 4.73; N, 4.38\%; Found: C, 73.28; H, 4.69; N, 4.28\%.

Synthesis of (Z)-4-((5-oxo-2-phenyl-1-(4-(5-aryl-4,5dihdro-1 $H$-pyrazol-3-yl)phenyl)-1H-imidazol-4(5H)-ylid ene)methyl)phenyl-4-methylbenzene sulfonate $\left(6_{\mathrm{a}-\mathrm{c}}\right)$.

General procedure

A mixture of the chalcones $\mathbf{5}_{\mathrm{a}-\mathrm{c}}(0.002 \mathrm{~mole})$ and hydrazine hydrate $(0.004 \mathrm{~mole})$ in ethanol $(20 \mathrm{ml})$ was refluxed for 6 hours. The solid that separated out was recrystallized from ethanol to give $\mathbf{6}_{\mathrm{a}-\mathrm{c}}$.

(Z)-4-((5-oxo-2-phenyl-1-(4-(5-phenyl-4,5-dihydro-1H-p yrazol-3-yl)phenyl)-1 $H$-imidazol-4 (5H)-ylidene) methyl) phenyl-4-methylbenzenesulfonate $6_{\mathbf{a}}$ :

Yellow crystals; Yield 43\%; m.p. $265-267^{\circ} \mathrm{C}$; IR (KBr): v/ $\mathrm{cm}^{-1}: 1670$ (CO, amidic), 3200-3260 (NH), $1360\left(\mathrm{SO}_{3}\right)$, $1600(\mathrm{C}=\mathrm{N})$; $\operatorname{EIMS}(\mathrm{m} / \mathrm{z})(\%): 638\left(\mathrm{M}^{+}, 32\right), 576(44), 480$ (54), 340 (100), 213 (23), 81 (15), 65 (34); ${ }^{1} \mathrm{H}$ NMR (DMSO) $(\delta, \mathrm{ppm}), 2.4\left(\mathrm{~s}, 3 \mathrm{H}, \mathrm{CH}_{3}\right), 3.1\left(\mathrm{~d}, 2 \mathrm{H}, \mathrm{CH}_{2}\right), 8.15(\mathrm{t}, 1 \mathrm{H}, \mathrm{CH})$, $9.5(\mathrm{~s}, 1 \mathrm{H},-\mathrm{NH}), 7.1-8.2(\mathrm{~m}, 23 \mathrm{H}, \mathrm{Ar}-\mathrm{H}, \mathrm{CH}=\mathrm{C})$; Anal. for $\mathrm{C}_{38} \mathrm{H}_{30} \mathrm{~N}_{4} \mathrm{O}_{4} \mathrm{~S}$ (638.7): Calcd.: C, 71.45; H, 4.73; N, 8.77\%; 
Found: C, 71.42; H, 4.69; N, 8.72\%.

(Z)-4-((1-(4-(5-(4-methoxyphenyl)-4,5-dihydro-1H-pyraz ol-3-yl)phenyl)-5-oxo-2-phenyl-1 $H$-imidazol-4(5H)-ylide ne)methyl)phenyl-4-methylbenzenesulfonate $6_{b}$ :

Yellow crystals; Yield 55\%; m.p. $210-212^{\circ} \mathrm{C}$; IR $(\mathrm{KBr})$ : $v / \mathrm{cm}^{-1}: 1670$ (CO, amidic), 3200-3260 (NH), $1360\left(\mathrm{SO}_{3}\right)$, $1600(\mathrm{C}=\mathrm{N})$; $\operatorname{EIMS}(\mathrm{m} / \mathrm{z})(\%): 668\left(\mathrm{M}^{+}, 25\right), 586(19), 430$ (45), 280 (100), 158 (18), 91 (27), 65 (37); ${ }^{1}$ H NMR (DMSO) $(\delta, \mathrm{ppm}), 2.4\left(\mathrm{~s}, 3 \mathrm{H}, \mathrm{CH}_{3}\right), 3.9\left(\mathrm{~s}, 3 \mathrm{H}, \mathrm{OCH}_{3}\right), 3.1(\mathrm{~d}, 2 \mathrm{H}$, $\left.\mathrm{CH}_{2}\right), 8.15(\mathrm{t}, 1 \mathrm{H}, \mathrm{CH}), 9.5(\mathrm{~s}, 1 \mathrm{H},-\mathrm{NH}), 7.1-8.2(\mathrm{~m}, 23 \mathrm{H}$, Ar- $\mathrm{H}, \mathrm{CH}=\mathrm{C})$; Anal. for $\mathrm{C}_{39} \mathrm{H}_{32} \mathrm{~N}_{4} \mathrm{O}_{5} \mathrm{~S}$ (668.7): Calcd.: $\mathrm{C}$, $70.04 ; \mathrm{H}, 4.82 ; \mathrm{N}, 8.37 \%$; Found: C, 70.0; H, 4.79; N, 8.38\%. (Z)-4-((5-oxo-2-phenyl-1-(4-(5-(p-tolyl)-4,5-dihydro-1 Hpyrazol-3-yl)phenyl)- $1 H$-imidazol-4-(5H)-ylidene) methyl)phenyl-4-methylbenzenesulfonate $\boldsymbol{6}_{\mathrm{c}}$ :

Yellow crystals; Yield $41 \%$; m.p. $180-182^{\circ} \mathrm{C}$; IR (KBr): $v / \mathrm{cm}^{-1}: 1670$ (CO, amidic), 3200-3260 (NH), $1360\left(\mathrm{SO}_{3}\right)$, $1600(\mathrm{C}=\mathrm{N})$; EIMS $(\mathrm{m} / \mathrm{z})(\%): 652\left(\mathrm{M}^{+}, 31\right), 550(45), 480$ (43), 323 (100), 148 (73), 91 (49), 65 (28); ${ }^{1}$ H NMR (DMSO) $(\delta, \mathrm{ppm}), 2.4\left(\mathrm{~s}, 3 \mathrm{H}, \mathrm{CH}_{3}\right), 2.1\left(\mathrm{~s}, 3 \mathrm{H}, \mathrm{CH}_{3}\right), 3.1\left(\mathrm{~d}, 2 \mathrm{H}, \mathrm{CH}_{2}\right)$, $8.15(\mathrm{t}, 1 \mathrm{H}, \mathrm{CH}), 9.5(\mathrm{~s}, 1 \mathrm{H},-\mathrm{NH}), 7.1-8.2(\mathrm{~m}, 23 \mathrm{H}, \mathrm{Ar}-\mathrm{H}$, $\mathrm{CH}=\mathrm{C}$ ); Anal. for $\mathrm{C}_{39} \mathrm{H}_{32} \mathrm{~N}_{4} \mathrm{O}_{4} \mathrm{~S}$ (652.7): Calcd.: $\mathrm{C}, 71.76 ; \mathrm{H}$, 4.94; N, 8.57\%; Found: C, 71.71; H, 4.90; N, 8.51\%.

Synthesis of (Z)-4-((1-(4-(5-cyano-6-oxo-4-aryl1,6-dihydropyridin-3-yl)phenyl)-5-oxo-2-phenyl-1 $H$-imidazol-4(5H)-ylidene)methyl)phenyl-4-methylbenzene sulfonate $\left(7_{\mathrm{a}-\mathrm{c}}\right)$.

General procedure

A mixture of compound $\mathbf{5}_{\mathrm{a}-\mathrm{c}}(0.01 \mathrm{~mole})$, ethylcyanoacetate $(0.01 \mathrm{~mole})$ and ammonium acetate $(0.08 \mathrm{~mole})$ was refluxed in ethanol $(30 \mathrm{ml})$ for 6 hours. The solid that separated after cooling was recrystallized from ethanol to give $7_{\mathrm{a}-\mathrm{c}}$.

(Z)-4-((1-(4-(5-cyano-6-oxo-4-phenyl-1,6-dihydropyridin -3-yl)phenyl)-5-oxo-2-phenyl-1 $H$-imidazol-4(5H)-ylidene )methyl)phenyl-4-methylbenzenesulfonate $7_{\mathrm{a}}$ :

Yellow crystals; Yield 36\%; m.p. $169-171^{\circ} \mathrm{C}$; IR ( $\left.\mathrm{KBr}\right)$ : $v / \mathrm{cm}^{-1}: 1680(\mathrm{CO}$, amidic), $3240(\mathrm{NH}), 1661(\mathrm{CONH}), 1360$ $\left(\mathrm{SO}_{3}\right), 1600(\mathrm{C}=\mathrm{N}), 2150(\mathrm{CN})$; EIMS $(\mathrm{m} / \mathrm{z})(\%): 688\left(\mathrm{M}^{+}\right.$, 21), 588 (26), 491 (36), 345 (100), 148 (53), 91 (47), 65 (34); Anal. for $\mathrm{C}_{41} \mathrm{H}_{28} \mathrm{~N}_{4} \mathrm{O}_{5} \mathrm{~S}(688.7)$ : Calcd.: C, 71.5; H, 4.1; N, $8.13 \%$; Found: C, $71.2 ; \mathrm{H}, 4.0 ; \mathrm{N}, 8.08 \%$.

(Z)-4-((1-(4-(5-cyano-4-(4-methoxyphenyl)-6-oxo-1,6-dih ydropyrdin-3-yl)phenyl)-5-oxo-2-phenyl-1H-imidazol-4 (5H)-ylidene)methyl)phenyl-4-methylbenzenesulfonate $7_{\mathrm{b}}$ :

Yellow crystals; Yield 43\%; m.p. $189-191^{\circ} \mathrm{C}$; IR ( $\mathrm{KBr}$ ): $v / \mathrm{cm}^{-1}: 1680(\mathrm{CO}$, amidic), $3240(\mathrm{NH}), 1661(\mathrm{CONH}), 1360$ $\left(\mathrm{SO}_{3}\right), 1600(\mathrm{C}=\mathrm{N}), 2150(\mathrm{CN})$; EIMS $(\mathrm{m} / \mathrm{z})(\%): 718\left(\mathrm{M}^{+}\right.$, 11), 666 (52), 591 (44), 432 (100), 280 (38), 151 (10), 65 (45); ${ }^{1} \mathrm{H}$ NMR (DMSO) $(\delta, \mathrm{ppm}), 2.4\left(\mathrm{~s}, 3 \mathrm{H}, \mathrm{CH}_{3}\right), 3.8(\mathrm{~s}, 3 \mathrm{H}$, $\left.\mathrm{OCH}_{3}\right), 6.5(\mathrm{~s}, 1 \mathrm{H}, \mathrm{CH}), 6.8-8.2(\mathrm{~m}, 24 \mathrm{H}, \mathrm{Ar}-\mathrm{H}, \mathrm{CH}=\mathrm{C}, \mathrm{NH})$; Anal. for $\mathrm{C}_{42} \mathrm{H}_{30} \mathrm{~N}_{4} \mathrm{O}_{6} \mathrm{~S}$ (718.7): Calcd.: $\mathrm{C}, 70.18 ; \mathrm{H}, 4.2 ; \mathrm{N}$, 7.79\%; Found: C, 70.11; H, 4.19; N,7.78\%. (Z)-4-((1-(4-(5-cyano-6-oxo-4-(p-tolyl)-1,6-dihydropyridi n-3-yl)phenyl)-5-oxo-2-phenyl-1 $H$-imidazol-4(5H)-yliden e)methyl)phenyl-4-methylbenzenesulfonate $7_{\mathfrak{c}}$ :

Yellow crystals; Yield 36\%; m.p. $155-157^{\circ} \mathrm{C}$; IR (KBr): $v / \mathrm{cm}^{-1}: 1680(\mathrm{CO}$, amidic), $3240(\mathrm{NH}), 1661(\mathrm{CONH}), 1360$ $\left(\mathrm{SO}_{3}\right), 1600(\mathrm{C}=\mathrm{N}), 2150(\mathrm{CN})$; EIMS $(\mathrm{m} / \mathrm{z})(\%): 702\left(\mathrm{M}^{+}\right.$, 24), 630 (24), 587 (54), 433 (100), 268 (43), 121 (11), 65 (38); ${ }^{1} \mathrm{H}$ NMR (DMSO) $(\delta, \mathrm{ppm}), 2.4\left(\mathrm{~s}, 3 \mathrm{H}, \mathrm{CH}_{3}\right), 2.1(\mathrm{~s}, 3 \mathrm{H}$, $\left.\mathrm{CH}_{3}\right), 6.5(\mathrm{~s}, 1 \mathrm{H}, \mathrm{CH}), 6.8-8.2(\mathrm{~m}, 24 \mathrm{H}, \mathrm{Ar}-\mathrm{H}, \mathrm{CH}=\mathrm{C}, \mathrm{NH})$; Anal. for $\mathrm{C}_{42} \mathrm{H}_{30} \mathrm{~N}_{4} \mathrm{O}_{5} \mathrm{~S}$ (702.7): Calcd.: C, 71.78; H, 4.3; N, 7.96\%; Found: C, 71.73; H, 4.3; N, 7.88\%.

Synthesis of (Z)-4-((1-(4-(5-cyano-6-imino-4-phenyl1,6-dihydropyridin-3-yl)phenyl)-5-oxo-2-phenyl-1Himidazol-4(5H)-ylidene)methyl)phenyl-4-methyl benzene sulfonate $\left(\mathbf{8}_{\mathrm{a}-\mathrm{c}}\right)$.

General procedure

A mixture of $\mathbf{5}_{\mathrm{a}-\mathrm{c}}(0.01 \mathrm{~mole})$, malononitrile $(0.012 \mathrm{~mole})$ and ammonium acetate $(0.08 \mathrm{~mole})$ was refluxed for 5 hours in ethanol $(30 \mathrm{ml})$. The reaction mixture was left to cool and poured into beaker containing onto ice-water. The solid that separated out was filtered off, washed with water and recrystallized several times methanol to give $\mathbf{8}_{\text {a-c }}$.

(Z)-4-((1-(4-(5-cyano-6-imino-4-phenyl-1,6-dihydropyrid in-3-yl)phenyl)-5-oxo-2-phenyl- $1 H$-imidazol-4(5H)-ylide ne)methyl)phenyl-4-methylbenzenesulfonate $8_{\mathrm{a}}$ :

Yellow crystals; Yield $43 \%$; m.p. $149-151^{\circ} \mathrm{C}$; IR (KBr): $v / \mathrm{cm}^{-1}: 1690$ (CO, amidic), 3279-3330 (2NH), $2100(\mathrm{CN})$, $1360\left(\mathrm{SO}_{3}\right), 1620(\mathrm{C}=\mathrm{N})$; EIMS $(\mathrm{m} / \mathrm{z})(\%): 687\left(\mathrm{M}^{+}, 32\right), 573$ (15), 477 (44), 340 (100), 228 (24), 131 (10), 65 (28); ${ }^{1} \mathrm{H}$ NMR (DMSO) $(\delta, \mathrm{ppm}), 2.4\left(\mathrm{~s}, 3 \mathrm{H}, \mathrm{CH}_{3}\right), 6.5(\mathrm{~s}, 1 \mathrm{H}, \mathrm{CH})$, 6.8-8.2 (m, 25H, Ar-H, $\mathrm{CH}=\mathrm{C}, \quad 2 \mathrm{NH})$; Anal. for $\mathrm{C}_{41} \mathrm{H}_{29} \mathrm{~N}_{5} \mathrm{O}_{4} \mathrm{~S}$ (687.7): Calcd.: C, 71.6; H, 4.25; N, 10.18\%; Found: C, 71.1; H, 4.22; N, 10.11\%.

(Z)-4-((1-(4-(5-cyano-6-imino-4-(4-methoxyphenyl)-1,6-d ihydropyrdin-3-yl)phenyl)-5-oxo-2-phenyl-1 $H$-imidazol $-4(5 H)$-ylidene)methyl)phenyl-4-methylbenzenesulfonate $8_{\mathrm{b}}$ :

Yellow crystals; Yield 49\%; m.p. $188-190^{\circ} \mathrm{C}$; IR (KBr): $v / \mathrm{cm}^{-1}: 1690(\mathrm{CO}$, amidic), 3279-3330 (2NH), $2100(\mathrm{CN})$, $1360\left(\mathrm{SO}_{3}\right), 1620(\mathrm{C}=\mathrm{N})$; EIMS $(\mathrm{m} / \mathrm{z})(\%): 717\left(\mathrm{M}^{+}, 23\right), 686$ (15), 567 (43), 472 (100), 278 (33), 161 (15), 65 (36); Anal. for $\mathrm{C}_{42} \mathrm{H}_{31} \mathrm{~N}_{5} \mathrm{O}_{5} \mathrm{~S}$ (717.79): Calcd.: C, 70.28; $\mathrm{H}, 4.357 ; \mathrm{N}$, 9.75\%; Found: C, 70.21; H, 4.32; N, 9.71\%.

(Z)-4-((1-(4-(5-cyano-6-imino-4-(p-tolyl)-1,6-dihydropyri din-3-yl)phenyl)-5-oxo-2-phenyl-1 $H$-imidazol-4(5H)-ylid ene)methyl)phenyl-4-methylbenzenesulfonate $8_{\mathrm{c}}$ :

Yellow crystals; Yield $39 \%$; m.p. $222-224^{\circ} \mathrm{C}$; IR (KBr): $v / \mathrm{cm}^{-1}: 1690(\mathrm{CO}$, amidic), 3279-3330 (2NH), $2100(\mathrm{CN})$, $1360\left(\mathrm{SO}_{3}\right), 1620(\mathrm{C}=\mathrm{N})$; EIMS $(\mathrm{m} / \mathrm{z})(\%): 701\left(\mathrm{M}^{+}, 32\right), 676$ (27), 545 (34), 462 (100), 278 (53), 181 (13), 65 (12); Anal. for $\mathrm{C}_{42} \mathrm{H}_{31} \mathrm{~N}_{5} \mathrm{O}_{4} \mathrm{~S}$ (701.79): Calcd.: $\mathrm{C}, 71.88 ; \mathrm{H}, 4.45 ; \quad \mathrm{N}$, 9.97\%; Found: C, 71.81; H, 4.42; N, 9.88\%.

Synthesis of (Z)-4-((5-oxo-1-(4-(3-oxobutanoyl) phenyl)-2-phenyl-1 $\mathrm{H}$-imidazol-4(5H)-ylidene)methyl) phenyl-4-methylbenzene sulfonate (9).

A suspension of compound 3 ( 0.01 mole) in freshly distilled ethylacetate $(0.5 \mathrm{~mole})$ was slowly added to powdered sodium metal $(0.2 \mathrm{~mole})$. The reaction mixture was refluxed for 8 hours, cooled then acidified with acetic acid. The precipitated solid product was separated and recrystallized from ethanol to give 9. 
Orange crystals; Yield 28\%; m.p. 206-208 ${ }^{\circ} \mathrm{C}$; IR ( $\left.\mathrm{KBr}\right)$ : $v / \mathrm{cm}^{-1}: 1682$ (CO, amidic), 1618-1622 (two CO), $1360\left(\mathrm{SO}_{3}\right)$, $1600(\mathrm{C}=\mathrm{N})$; $\operatorname{EIMS}(\mathrm{m} / \mathrm{z})(\%): 577\left(\mathrm{M}^{+}-1,26\right), 426(70), 392$ (29), 342 (42), 224 (50), 169 (92), 131 (100), 80 (84); Anal. for $\mathrm{C}_{33} \mathrm{H}_{26} \mathrm{~N}_{2} \mathrm{O}_{6} \mathrm{~S}$ (578.6): Calcd.: C, 68.5; H, 4.53; N, $4.84 \%$; Found: C, 68.1; H, 4.51; N, $4.79 \%$.

Synthesis of (Z)-4-((1-(4-(6-methyl-2-thioxo-2,5-dihydro pyrimidin-4-yl)phenyl)-5-oxo-2-phenyl- $1 \mathrm{H}$-imidazol-4(5 $H$ )-ylidene)methyl)phenyl-4-methylbenzenesulfonate (10).

A mixture of compound 9 (0.002mole) and thiourea $(0.01 \mathrm{~mole})$ in ethanol $(30 \mathrm{ml})$ was refluxed for 6 hours. The reaction mixture was left to cool then poured onto ice-cold water, filtered off and recrystallized from ethanol to give $\mathbf{1 0}$. Yellow powder; Yield $61 \%$; m.p. $199-201{ }^{\circ} \mathrm{C}$; IR ( $\mathrm{KBr}$ ): $\mathrm{v} / \mathrm{cm}^{-1}: 1680$ (CO, amidic), $1370(\mathrm{C}=\mathrm{S}), 1360\left(\mathrm{SO}_{3}\right), 1600$ $(\mathrm{C}=\mathrm{N})$; $\operatorname{EIMS}(\mathrm{m} / \mathrm{z})(\%): 621\left(\mathrm{M}^{+}+2,17\right), 536(56), 450(11)$, 306 (18), 263 (16), 143 (16), 105 (83), 91 (100); ${ }^{1} \mathrm{H}$ NMR (DMSO) ( $\delta$, ppm), $2.4\left(\mathrm{~s}, 3 \mathrm{H}, \mathrm{CH}_{3}\right), 1.3\left(\mathrm{~s}, 2 \mathrm{H}, \mathrm{CH}_{2}\right), 1.9(\mathrm{~s}$, $\left.3 \mathrm{H}, \mathrm{CH}_{3}\right), 6.8-8.2(\mathrm{~m}, 18 \mathrm{H}, \mathrm{Ar}-\mathrm{H}, \mathrm{CH}=\mathrm{C})$; Anal. for $\mathrm{C}_{34} \mathrm{H}_{26} \mathrm{~N}_{4} \mathrm{O}_{4} \mathrm{~S}_{2}(618.7)$ : Calcd.: C, 66.0; H, 4.24; $\mathrm{N} 9.05 \%$; Found: C, 59.94; H, 4.20; N, 9.00\%.

Synthesis of (Z)-ethyl 4-(5-oxo-2-phenyl-4-(4-(tosyloxy) benzylidene)-4,5-dihydro-1 $H$-imidazol-1-yl)benzoate (11).

A mixtuure of oxazolone $2(0.01 \mathrm{~mole})$ and ethyl $p$-amino benzoate $(0.01 \mathrm{~mole})$ in glacial acetic acid $(30 \mathrm{ml})$ containing freshly fused sodium acetate $(0.5 \mathrm{gm})$ was heated under reflux for 10 hours. The reaction mixture was concentrated under reduced pressure and then poured onto ice-cold water. The separated solid was filtered off, and recrystallized from acetone to give $\mathbf{1 1}$.

Yellow crystals; Yield $44 \%$; m.p. $150-152^{\circ} \mathrm{C}$; IR (KBr): $v / \mathrm{cm}^{-1}: 1680$ (CO, amidic), 1725 (ester, $\left.\mathrm{CO}\right), 1360\left(\mathrm{SO}_{3}\right)$, $1644(\mathrm{C}=\mathrm{N})$; EIMS $(\mathrm{m} / \mathrm{z})(\%): 566\left(\mathrm{M}^{+}, 27\right), 476(39), 395$ (15), 344 (27), 263 (8), 145 (52), 78 (24), 43 (100); ${ }^{1} \mathrm{H}$ NMR (DMSO) $(\delta, \mathrm{ppm}), 2.4\left(\mathrm{~s}, 3 \mathrm{H}, \mathrm{CH}_{3}\right), 4.39-4.42(\mathrm{q}, 2 \mathrm{H}$, $\left.\mathrm{CH}_{2} \mathrm{CH}_{3}\right), 1.39-1.43\left(\mathrm{t}, 3 \mathrm{H}, \mathrm{CH}_{2} \mathrm{CH}_{3}\right), 7.1-8.2(\mathrm{~m}, 18 \mathrm{H}, \mathrm{Ar}-\mathrm{H}$, $\mathrm{CH}=\mathrm{C}$ ); Anal. for $\mathrm{C}_{32} \mathrm{H}_{26} \mathrm{~N}_{2} \mathrm{O}_{6} \mathrm{~S}(566.6)$ : Calcd.: $\mathrm{C}, 67.83 ; \mathrm{H}$, 4.63; N, 4.94\%; Found: C, 67.78; H, 4.61; N, 4.88\%.

Synthesis of (Z)-4-((1-(4-(benzylcarbamoyl)phenyl) -5-oxo-2-phenyl-1 $H$-imidazol-4(5H)-ylidene)methyl)phe nyl-4-methylbenzenesulfonate (12).

A mixture of compound $\mathbf{1 1}(0.01 \mathrm{~mole})$ and benzylamine $(0.01 \mathrm{~mole})$ was heated at $150^{\circ} \mathrm{C}$ in an oil bath for 6 hours, then cooled. The solid that separated was washed with water and recrystallized from methanol to give $\mathbf{1 2}$.

Brown powder; Yield 33\%; m.p. $190-192^{\circ} \mathrm{C}$; IR (KBr): $v / \mathrm{cm}^{-1}: 1690\left(\mathrm{CO}\right.$, amidic), $3478(\mathrm{NH}), 1360\left(\mathrm{SO}_{3}\right), 1633$ $(\mathrm{C}=\mathrm{N})$; EIMS $(\mathrm{m} / \mathrm{z})(\%): 626.6\left(\mathrm{M}^{+}-1,10.9\right), 403(10), 248$ (19), 224 (100), 145 (18), 118 (38), 106 (14), 91 (29); ${ }^{1} \mathrm{H}$ NMR (DMSO) $(\delta, \mathrm{ppm}), 2.4\left(\mathrm{~s}, 3 \mathrm{H}, \mathrm{CH}_{3}\right), 4.2(\mathrm{~s}, 2 \mathrm{H}$, $\mathrm{CH}_{2} \mathrm{Ph}$ ), 7.1-8.3 (m, 24H, Ar-H, $\left.\mathrm{CH}=\mathrm{C}, \mathrm{NH}\right)$; Anal. for $\mathrm{C}_{37} \mathrm{H}_{29} \mathrm{~N}_{3} \mathrm{O}_{5} \mathrm{~S}$ (627.7): Calcd.: $\mathrm{C}, 70.8 ; \mathrm{H}, 4.66 ; \mathrm{N}, 6.69 \%$; Found: C, 70.2; H, 4.63; N, 6.68\%.

Reaction of imidazolone (11) with hydrazine hydrate.

A mixture of imidazolone $\mathbf{1 1}(0.02 \mathrm{~mole})$, and hydrazine hydrate $(0.06 \mathrm{~mol})$ in ethanol $(50 \mathrm{ml})$ was heated under reflux for 12 hours. The reaction mixture was concentrated under reduced pressure and then poured onto ice-cold water. The solid that separated was filtered off, and dried.

(8gm) of these solid products were subjected to a column chromatography using silica gel as an adsorbent. Elution of the column was performed using two different ratios of petroleum ether/ethylacetate.

Fraction 1: This was eluted by petroleum ether/ ethylacetate (3:1) as eluent from column afforded a pure compound (13)

Ethyl-4-((4Z)-5-hydrazono-2-phenyl-4-(4-(tosyloxy)benz ylidene)-4,5-dihydro- $1 H$-imidazol-1-yl)benzoate (13).

White crystals; Yield $25 \%$; m.p. $251-253^{\circ} \mathrm{C}$; IR (KBr): $v / \mathrm{cm}^{-1}: 1724$ (ester, $\left.\mathrm{CO}\right), 3411-3460\left(\mathrm{NH}_{2}\right), 1370\left(\mathrm{SO}_{3}\right)$, $1637(\mathrm{C}=\mathrm{N})$; $\operatorname{EIMS}(\mathrm{m} / \mathrm{z})(\%): 580\left(\mathrm{M}^{+}, 47\right), 429(32), 349$ (59), 293 (65), 213 (39), 177 (34), 137 (78), 119 (24), 61 (100); ${ }^{1} \mathrm{H}$ NMR (DMSO) $(\delta, \mathrm{ppm}), 2.4\left(\mathrm{~s}, 3 \mathrm{H}, \mathrm{CH}_{3}\right)$, 4.31-4.35 (q, $2 \mathrm{H}, \mathrm{CH}_{2}$ ), 1.2-1.3 (t, $\left.3 \mathrm{H}, \mathrm{CH}_{3}\right), 6.5(\mathrm{br}, 2 \mathrm{H}$, $\left.\mathrm{NH}_{2}\right), 7.1-8.3 \quad(\mathrm{~m}, \quad 18 \mathrm{H}, \quad \mathrm{Ar}-\mathrm{H}, \quad \mathrm{CH}=\mathrm{C}) ; \quad$ Anal. for $\mathrm{C}_{32} \mathrm{H}_{28} \mathrm{~N}_{4} \mathrm{O}_{5} \mathrm{~S}$ (580.6): Calcd.: C, 66.19; H, 4.86; N, $9.65 \%$; Found: C, 66.11; H, 4.80; N, $9.55 \%$.

Fraction 2: This was eluted by petroleum ether/ ethylacetate (3:1) as eluent from column afforded a pure compound (14).

(Z)-4-((6-0xo-3-phenyl-1,6-dihydro-1,2,4-triazin-5(2H)-y lidene)methyl)phenyl-4-methyl benzenesulfonate (14).

White crystals; Yield $15 \%$; m.p. $111-113^{\circ} \mathrm{C}$; IR $(\mathrm{KBr})$ : $v / \mathrm{cm}^{-1}: 1686$ (CO, amidic), 3282-3315 (2NH), $1360\left(\mathrm{SO}_{3}\right)$, $1645(\mathrm{C}=\mathrm{N})$; EIMS $(\mathrm{m} / \mathrm{z})(\%): 432.3\left(\mathrm{M}^{+}-1,46\right), 371(100)$, 225 (50), 189 (50), 127 (60), 110 (88), 85 (52), 56 (96); ${ }^{1} \mathrm{H}$ NMR (DMSO) $(\delta, \mathrm{ppm}), 2.4\left(\mathrm{~s}, 3 \mathrm{H}, \mathrm{CH}_{3}\right), 4.2(\mathrm{br}, \mathrm{H}, \mathrm{NH})$, 7.2-8.2 (m, 15H, Ar-H, CH=C, NHCO); Anal. for $\mathrm{C}_{23} \mathrm{H}_{19} \mathrm{~N}_{3} \mathrm{O}_{4} \mathrm{~S}$ (433.4): Calcd.: C, 63.73; H, 4.42; N, $9.69 \%$; Found: C, 63.71; H, 4.40; N, $9.65 \%$.

Fraction 3: This was eluted by petroleum ether/ ethylacetate (3:3) as eluent from column afforded a pure compound (15).

(Z)-4-((1-(4(hydrazinecarbonyl)phenyl)5-hydrazinyl-2-p henyl-1 $H$-imidazol-4(5H)-ylidene)methyl)phenyl-4-meth -ylbenzenesulfonate (15).

White crystals; Yield $21 \%$; m.p. $134-136^{\circ} \mathrm{C}$; IR $(\mathrm{KBr})$ : $v / \mathrm{cm}^{-1}: 1652$ (CO, amidic), $3430(\mathrm{NH}), 3268-3380\left(2 \mathrm{NH}_{2}\right)$, $1360\left(\mathrm{SO}_{3}\right), 1640(\mathrm{C}=\mathrm{N})$; $\operatorname{EIMS}(\mathrm{m} / \mathrm{z})(\%): 567\left(\mathrm{M}^{+}, 21\right), 481$ (10), 411 (100), 339 (75), 247 (16), 224 (57), 179 (11), 105 (87); ${ }^{1} \mathrm{H}$ NMR (DMSO) ( $\delta$, ppm), $2.4\left(\mathrm{~s}, 3 \mathrm{H}, \mathrm{CH}_{3}\right), 9.1(\mathrm{~s}, 1 \mathrm{H}$, NHCO), 7.1-8.3 (m, $\left.22 \mathrm{H}, \mathrm{Ar}-\mathrm{H}, \mathrm{CH}=\mathrm{C}, 2 \mathrm{NH}_{2}\right)$; Anal. for $\mathrm{C}_{30} \mathrm{H}_{27} \mathrm{~N}_{6} \mathrm{O}_{4} \mathrm{~S}$ (567.6): Calcd.: C, 63.48; H, 4.79; N, $14.81 \%$; Found: C, 63.41; H, $4.80 ; \mathrm{N}, 14.75 \%$.

Fraction 4: This was eluted by petroleum ether/ ethylacetate $(3: 2)$ as eluent from column afforded a pure compound (16).

(Z)-4-((1-(4-(hydrazinecarbonyl)phenyl)-5-oxo-2-phenyl$1 H$-imidazol-4(5H)-ylidene)methyl)phenyl-4-methyl benzenesulfonate (16).

White crystals; Yield $33 \%$; m.p. $141-143^{\circ} \mathrm{C}$; IR $(\mathrm{KBr})$ : $v / \mathrm{cm}^{-1}: 1690\left(\mathrm{CO}\right.$, amidic), $3460(\mathrm{NH}), 3407-3469\left(\mathrm{NH}_{2}\right)$, 
$1370\left(\mathrm{SO}_{3}\right), 1644(\mathrm{C}=\mathrm{N}) ; \operatorname{EIMS}(\mathrm{m} / \mathrm{z})(\%): 551.6\left(\mathrm{M}^{+}-1,17\right)$, 518 (14), 427 (28), 306 (11), 244 (39), 189 (53), 104 (70), 63 (100); ${ }^{1} \mathrm{H}$ NMR (DMSO) $(\delta, \mathrm{ppm}), 2.4\left(\mathrm{~s}, 3 \mathrm{H}, \mathrm{CH}_{3}\right), 12.2(\mathrm{~s}$, $1 \mathrm{H}, \mathrm{NHCO})$, 6.9-8.6 (m, 20H, $\left.\mathrm{Ar}-\mathrm{H}, \mathrm{CH}=\mathrm{C}, \mathrm{NH}_{2}\right)$; Anal. for $\mathrm{C}_{30} \mathrm{H}_{24} \mathrm{~N}_{4} \mathrm{O}_{5} \mathrm{~S}$ (552.6): Calcd.: C, 65.2; H, 4.38; N, $10.14 \%$; Found: C, 65.1; H, 4.33; N, $10.15 \%$.

Synthesis of 4-(2-benzamido-2-(4-oxo-4H-benzo [d] [1,3]0 xazin-2-yl)vinyl)phenyl-4-methylbenzenesulfonate (17).

A mixture of oxazolone $2(0.05 \mathrm{~mole})$ and anthranilic acid $(0.05$ mole $)$ in acetic acid was heated under reflux for 14 hours. The product obtained after cooling was filtered off and recrystallized from acetic acid to give $\mathbf{1 7 .}$

Yellow crystals; Yield $70 \%$; m.p. $190-192^{\circ} \mathrm{C}$; IR (KBr): $v / \mathrm{cm}^{-1}: 1760$ (CO, lactone), $3470(\mathrm{NH}), 1360\left(\mathrm{SO}_{3}\right), 1595$ $(\mathrm{C}=\mathrm{C}), 1640(\mathrm{C}=\mathrm{N})$; EIMS $(\mathrm{m} / \mathrm{z})(\%): 538\left(\mathrm{M}^{+}, 16\right), 383(35)$, 275 (100), 224 (54), 155 (42), 119 (15), 91 (97); ${ }^{1} \mathrm{HNMR}$ (DMSO) $(\delta, \mathrm{ppm}), 2.4\left(\mathrm{~s}, 3 \mathrm{H}, \mathrm{CH}_{3}\right), 4.9(\mathrm{~s}, 1 \mathrm{H}, \mathrm{CH}=\mathrm{C})$, 7.1-8.3 (m, $19 \mathrm{H}, \quad \mathrm{Ar}-\mathrm{H}, \quad \mathrm{CH}=\mathrm{C}, \mathrm{NHCO})$; Anal. for $\mathrm{C}_{30} \mathrm{H}_{22} \mathrm{~N}_{2} \mathrm{O}_{6} \mathrm{~S}$ (538.5): Calcd.: C, 66.9; H, 4.12; N, 5.19\%; Found: C, 66.4; H, 4.1; N, 5.18\%.

Microbiological procedures for the activity study.

Materials and method:

Media: Nutrient agar and Potato Dextrose Agar plates were used for bacterial and fungal organisms respectively.

Preparation of microbial suspension: The bacterial and fungal strains were subculture at $37^{\circ} \mathrm{C}$ for six hrs in the corresponding medium of three successive days. These suspensions were used to insulate the antibiograms.

Preparation of the biograms: The agar disk diffusion method was performed on each of the tested substance solution in dimethylformamide. Filter paper discs were impregnated with $1 \mathrm{ml}$ of the solution and placed on the inoculated plates. These plates after standing at $4^{\circ} \mathrm{C}$ for 2 hours were incubated at $37^{\circ} \mathrm{C}$ for 24 hours. The diameters of the inhibition zones were measured in millimeters.

\section{Conclusions}

A series of novel substituted imidazolone derivatives were synthesized by the reaction of oxazolone derivative 2 with some primary aromatic amines namely, $p$ - aminoacetophenone, ethyl $p$-aminobenzoate and anthranilic acid. All the compounds were subjected to biological screening. Most of compounds exhibited good activities, where $\mathbf{3}, \mathbf{5}_{\mathrm{b}}, \mathbf{5}_{\mathbf{c}}, \mathbf{6}_{\mathrm{a}}$, $7_{\mathfrak{b}}, 7_{\mathfrak{c}}, \mathbf{8}_{\mathfrak{a}}, \mathbf{9}, \mathbf{1 1}, 13$ and $\mathbf{1 4}$ exhibited good activities against Bacillus Thuringenesis and Klebseilla Pneumonia, while compounds $3,4, \mathbf{6}_{\mathrm{a}}, \mathbf{6}_{\mathrm{b}}, \mathbf{6}_{\mathrm{c}}, \mathbf{7}_{\mathfrak{c}}, \mathbf{8}_{\mathrm{a}}, \mathbf{8}_{\mathrm{c}}, \mathbf{1 1}, 12$ and 16 exhibited good activities against Trichoderma Herzianum and Trichoderma Virdi. This proves the high therapeutic value of these compounds and encourages further study to explore their biological potential.

\section{REFERENCES}

[1] Jaemin, L.; Takeshi,Y.; Hirofumi, K.; Makoto, K. Autonomic Neuroscience, 2012, 166, 72-76. TRPV1 expressing extrinsic primary sensory neurons play a protective role in mouse oxazolone-induced colitis.

[2] Abdel-Aty, A. S. World Journal of Agricultural Sciences 2009, 5, 105-113. Pesticidal Effects of Some Imidazolidine and Oxazolone Derivatives.

[3] Huan, Q.; Haiqin, L.; Hua-Ji, Q.; Valery, P.; Aihua, L. Journal of Molecular biology. 2012, 417, 129-143. Phagemid Vectors for Phage Display: Properties, Characteristics and Construction.

[4] Matthias, A.; Engel, M. K.; Norbert, S.; Sonja, M.; Mueller, T.; Winfried, L. N.; Markus, F. N.; Christoph, B.; Peter, W. R. Digestive and Liver Disease, 2012, 44, 24-29. Opposite effects of substance $\mathrm{P}$ and calcitonin gene-related peptide in oxazolone colitis.

[5] Habib, O. M.; Girges, M. M.; Moawad, E. B.; El-Shafei, A. M. Boll. Chim. Farmaceutico 1995, 134, 209-215. Synthetic approaches and biological evaluation of some new sulfonate ester-containing quinazoline derivatives as potentially active antimicrobial agents.

[6] Joshi, H.; Upadhyay, P.; Karia, D.; Baxi, A. J. European Journal of Medicinal Chemistry 2003, 38, 837-840. Synthesis of Some Novel Imidazolinones as Potent Anticonvulsant Agents.

[7] Mistry, R. N.; Desai, K. R. E-Journal of Chem. 2005, 2, 42-51. Studies on Synthesis of Some Novel Heterocyclic Azlactone Derivatives and Imidazolinone Derivatives and their Antimicrobial Activit.

[8] Siddiqui, S. A.; Bhusare, S. R.; Jarikote, D. V.; Pawar, R.; Vibhute, P. Y. B. Bull. Korean Chem. Soc., 2001, 22, 1033-1036. New Novel Synthesis and Antibacterial Activity of 1-(Substituted phenyl)-2-phenyl-4-(3-halo, 4-hydroxy 5-methoxy benzylidene)-imidazole-5-ones. 